\title{
Fluid extraction from porous media by a slender permeable prolate-spheroid
}

\author{
Kang Ping Chen \\ School for Engineering of Matter, Transport and Energy \\ Arizona State University, Tempe, AZ 85287-6106, USA
}

\begin{abstract}
Fluid extraction from porous media by a slender permeable prolate-spheroid is analyzed. It is found that the flux density along the spheroid-reservoir surface increases with the sharpness of the spheroid tip; and the fluid production rate is significantly higher than that predicted by the cylinder model used in the literature. It is shown that the flow in the reservoir is a superposition of a confocal flow and a redistributive flow, with only the confocal flow being productive. For high spheroid-to-reservoir permeability ratios, there exists an optimum length-to-radius ratio that maximizes fluid production rate for a given spheroid body volume.
\end{abstract}

Email: k.p.chen@asu.edu; Tel: (480)965-0907; Fax: (480)727-9321

\section{Introduction}

Extraction of fluid stored in a porous medium is a problem of interest to hydrology, agriculture, plant biology, petroleum engineering and geothermal exploitation. Recent success of petroleum production from shale formation where the permeability can be as low as one nanoDarcy is an astonishing manifestation of the effectiveness of using hydraulic fractures for such fluid extractions. Meanwhile, the Arizona Sonora desert plants also possess root systems for efficient water-uptake from soil that ensure their survivals in a low annual rainfall and dry 
environment. Understanding what makes these men-made and natural structures efficient for fluid extraction from porous media has enormous implications for engineering, ecology and the earth's environment.

The mechanisms for the enhanced fluid production from hydraulically fractured wells have been historically attributed to two factors: a hydraulic fracture creates a large contact area with the fluid-bearing reservoir; and the fracture provides a high conductivity pathway for the fluid to flow easily to the well [1]. Recently, we have performed an analysis of the Darcy flow pattern in the reservoir and the flux density distribution along the fracture-reservoir surface, using a newly obtained analytical solution for finite fracture conductivities [2-3]. It is found that when the well pressure is lowered, the induced-flow in the reservoir develops a converging pattern that focuses the fluid to the fracture tip, creating a nearly singular flux density around the fracture tip. Increasing the sharpness of the fracture tip can promote the flux density distribution along the entire fracture-reservoir surface, which should be considered as an additional mechanism for enhancing fluid production.

Water-uptake by a single plant root bears similarities to fluid production from a fracture: a plant root resembles a slender prolate-spheroid with a large length-to-radius ratio and a converging tip. Thus, a root can be considered as the axisymmetric counterpart to a twodimensional fracture. However, there is a significant difference between a fracture and a root: when the tip sharpness is increased, the fluid-producing area of a fracture remains constant due to its two-dimensionality; while the fluid-producing area of a root's surface shrinks to zero in response to increased tip sharpness. Thus, there must be an optimum length-to-radius ratio that 
maximizes a root's water-uptake. Indeed there is discussion in the literature about tradeoffs between water-uptake and root length utilization [4], which is likely closely related to this length optimization issue.

At the macroscopic root system level in plant water-uptake studies [5-12], water-uptake by a plant root is represented by a sink term in the water mass balance equation in the soil [7]. This sink term, also called the water extraction function [9], represents water-uptake averaged over a large number of rootlets. This volumetric rate of uptake expressed as a sink is at the heart of all macroscopic models, and it must be derived from an analysis of the uptake at the mesoscopic single root scale. At the mesoscopic scale, a single root has been historically modeled geometrically as a long cylinder with a constant radius, drawing water from the soil in a cylindrical shell $[7,12-16]$. Water in the soil has been modeled to flow in the radial direction only, perpendicular to the cylinder's axis of symmetry. However, such a purely radial flow occurs only when the cylinder (root) is infinitely long, and this radial flow is inherently twodimensional. This commonly used model has limitations when applied to a realistic root, since all roots have a finite length and the flow around the end of a finite-length cylinder (root tip) is always three-dimensional. An outcome of neglecting this three-dimensional tip effect is the underestimate of the water flux density on the root-soil surface, which in turn results in an underestimate of the water-uptake by a single root.

In this work, we present an analytical solution for steady fluid extraction from a porous medium by a slender permeable prolate-spheroid, and we analyze the fluid production characteristics. The work focuses on tip effect and shape optimization for enhanced water-uptake. 
Understanding of this simple model lays the foundation for future development of more sophisticated models that incorporate such factors as complex root internal structure; coupling between water-uptake and nutrient-uptake; unsaturated soil conditions; and the unsteadiness of the uptake process.

\section{Mathematical formulation}

Consider fluid extraction from a fluid-saturated porous medium (reservoir) by a slender permeable prolate-spheroid. The reservoir has a permeability $\kappa_{s}$; and gravitational effect can be neglected. The prolate-spheroid is porous, with a uniform internal structure and an isotropic permeability $\kappa_{r}$ (Fig. 1). In a prolate spheroidal coordinates $(\tilde{\xi}, \tilde{\eta}, \tilde{\varphi})$, the prolate-spheroid surface is described by $\tilde{\xi}=\tilde{\xi}_{0}$. The Cartesian coordinates $(x, y, z)$ is related to the prolate spheroidal coordinates by

$$
x=L \cosh \tilde{\xi} \cos \tilde{\eta}, y=L \sinh \tilde{\xi} \sin \tilde{\eta} \sin \tilde{\varphi}, z=L \sinh \tilde{\xi} \sin \tilde{\eta} \cos \tilde{\varphi},
$$

where $L$ is the focal distance, $x_{0}=L \cosh \tilde{\xi}_{0}$ is the half-length of the body, which is also the distance from the base to the tip; and the radius at the base is $z_{0}=L \sinh \tilde{\xi}_{0}$. Because of symmetry, only half of the spheroid needs to be considered and the domain of interest is $0 \leq \tilde{\xi}<\infty, 0 \leq \tilde{\eta} \leq \pi / 2,0 \leq \tilde{\varphi} \leq 2 \pi$.

The movement of the fluid obeys Darcy's law; thus the fluid pressure $p$ in the reservoir satisfies the Laplace's equn., which for axisymmetric case can be written as

$$
\frac{\partial}{\partial \xi}\left[\left(\xi^{2}-1\right) \frac{\partial p}{\partial \xi}\right]+\frac{\partial}{\partial \eta}\left[\left(1-\eta^{2}\right) \frac{\partial p}{\partial \eta}\right]=0
$$


where $\xi=\cosh \tilde{\xi}, \eta=\cos \tilde{\eta}, \varphi=\tilde{\varphi}$, and $\cosh \tilde{\xi}_{0}=\xi_{0} \leq \xi<\infty, 0 \leq \eta \leq 1,0 \leq \varphi \leq 2 \pi$.

The inside of the prolate-spheroid is defined by $0 \leq \tilde{\xi} \leq \tilde{\xi}_{0}, 0 \leq \tilde{\eta} \leq \pi / 2,0 \leq \tilde{\varphi} \leq 2 \pi$, and the flow is predominantly along the x-direction for a slender body. The cross-sectional-areaaveraged pressure inside the body is

$$
p_{I}=\frac{\int_{0}^{\xi_{0}} p \sinh \tilde{\xi} d \tilde{\xi}}{\cosh \tilde{\xi}_{0}-1} .
$$

Using the symmetry condition along the x-axis and continuity of the flux density at the bodyreservoir surface, the fluid continuity eqn. inside the spheroid can be integrated across the crosssectional area to obtain a relation between the axial flux along the $\mathrm{x}$-direction inside the spheroid $q_{r}(\tilde{\eta})$ and flux entering the spheroid through the spheroid-reservoir surface, $q_{s}(\tilde{\eta})$ :

$$
\frac{\partial q_{r}}{\partial \tilde{\eta}}=2 \pi q_{s}(\tilde{\eta}) L^{2} \sinh \tilde{\xi}_{0} \sin \tilde{\eta} \sqrt{\sinh ^{2} \tilde{\xi}_{0}+\sin ^{2} \tilde{\eta}}
$$

where

$$
q_{s}(\tilde{\eta})=\left.\left(-\mathbf{i}_{\tilde{\xi}}\right) \bullet \mathbf{v}(\tilde{\xi}, \tilde{\eta})\right|_{\tilde{\xi}_{0}}=\left.\frac{\kappa_{s}}{\mu} \frac{1}{L \sqrt{\sinh ^{2} \tilde{\xi}+\sin ^{2} \tilde{\eta}}} \frac{\partial p}{\partial \tilde{\xi}}\right|_{\tilde{\xi}_{0}}
$$

Eqn. (4) can be written as

$$
C_{r t} \frac{d}{d \tilde{\eta}}\left(\sin \tilde{\eta} \frac{d p_{I}}{d \tilde{\eta}}\right)+\left.\sin \tilde{\eta} \frac{\partial p}{\partial \xi}\right|_{\xi_{0}}=0
$$

with the dimensionless conductivity $C_{r t}$ defined by

$$
\begin{aligned}
& C_{r t}=\frac{\kappa_{r}}{\kappa_{s}} \frac{\cosh \tilde{\xi}_{0}-1}{\sinh ^{2} \tilde{\xi}_{0}}=\frac{\lambda}{\xi_{0}+1}, \\
& \lambda=\frac{\kappa_{r}}{\kappa_{s}}, \xi_{0}=\cosh \tilde{\xi}_{0} .
\end{aligned}
$$


The pressure in the reservoir and the spheroid can be obtained by solving eqns. (2) and (6) with the condition of continuity in pressure and flux density at the spheroid-reservoir surface, as well as the boundary conditions:

$$
\begin{aligned}
& \xi=\xi_{e}=\cosh \tilde{\xi}_{e}: p=p_{e}(\text { outer boundary }) \\
& \tilde{\eta}=0: \quad \frac{d p_{I}}{d \tilde{\eta}}=0(\text { symmetry }) \\
& \tilde{\eta}=\pi / 2: p_{I}=p_{w}(\text { given well pressure })
\end{aligned}
$$

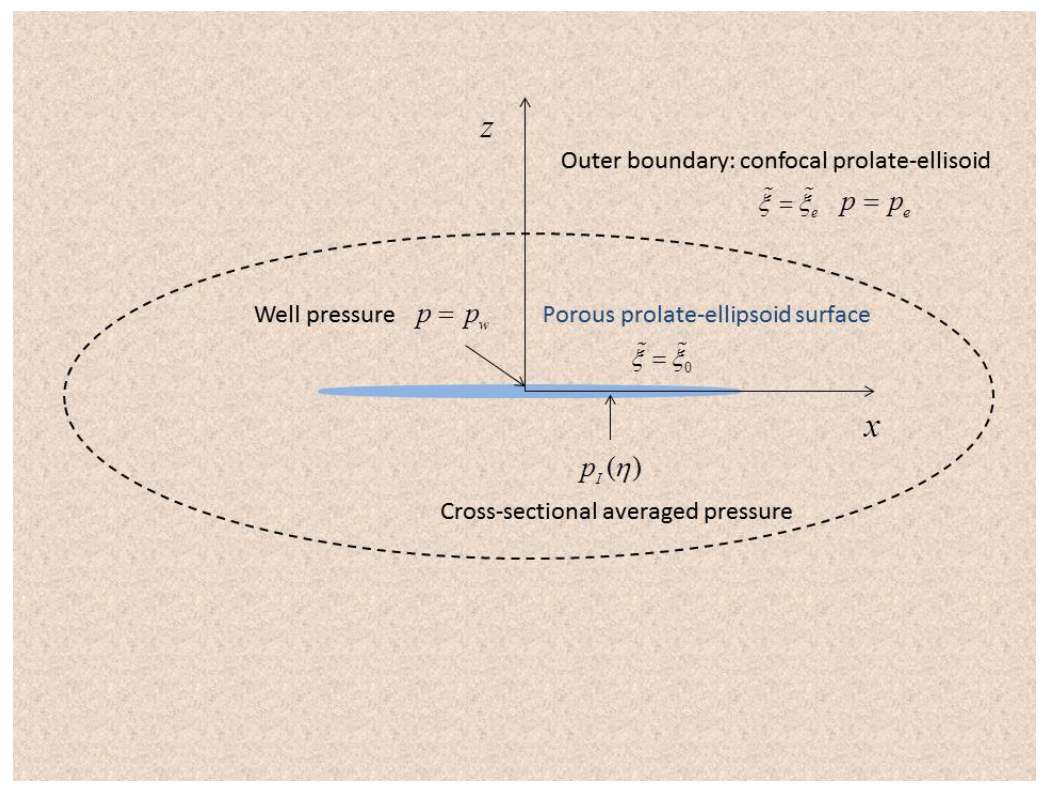

Fig. 1 Fluid extraction by a porous prolate-spheroid from a large reservoir.

\section{The pressure field and fluid production rate}

The pressure distribution in the reservoir is given by

$$
p(\xi, \eta)=\sum_{m=0}^{\infty}\left[A_{2 m} P_{2 m}(\xi)+B_{2 m} Q_{2 m}(\xi)\right] P_{2 m}(\eta), 1 \leq \xi_{0} \leq \xi<\infty, 0 \leq \eta \leq 1
$$

where $P_{2 m}$ and $Q_{2 m}$ are the zeroth-order associated Legendre function of the first and the second kind, respectively. The average pressure inside the prolate-spheroid is given by 


$$
\begin{aligned}
p_{I}(\eta)=p_{w} & -\frac{1}{C_{r t}} \sum_{m=1}^{\infty} \frac{A_{2 m} P_{2 m}^{\prime}\left(\xi_{0}\right)+B_{2 m} Q_{2 m}^{\prime}\left(\xi_{0}\right)}{2 m(2 m+1)} P_{2 m}(0)+\frac{B_{0} Q_{0}^{\prime}\left(\xi_{0}\right)}{C_{r t}} \ln (1+\eta) \\
& +\frac{1}{C_{r t}} \sum_{m=1}^{\infty} \frac{A_{2 m} P_{2 m}^{\prime}\left(\xi_{0}\right)+B_{2 m} Q_{2 m}^{\prime}\left(\xi_{0}\right)}{2 m(2 m+1)} P_{2 m}(\eta)
\end{aligned},
$$

where primes stand for derivatives. The coefficients $\left\{A_{2 m}, B_{2 m}\right\}$ are given in the Appendix.

The fluid production rate from one-half of the prolate-spheroid is

$$
Q=-\frac{2 \pi \kappa_{s} L}{\mu} B_{0}=\frac{2 \pi \kappa_{s} \Delta p L}{\mu} \frac{\xi_{0}^{2}-1}{H\left(\lambda, \xi_{0}, \xi_{e}\right)},
$$

where $\Delta p=p_{e}-p_{w}$ and

$$
\begin{aligned}
& H\left(\lambda, \xi_{0}, \xi_{e}\right)=\left(\xi_{0}^{2}-1\right)\left(Q_{0}\left(\xi_{0}\right)-Q_{0}\left(\xi_{e}\right)\right)+\frac{2 \ln 2-1-\mathrm{g}\left(\lambda, \xi_{0}, \xi_{e}\right)}{C_{r t}} ; \\
& g\left(\lambda, \xi_{0}, \xi_{e}\right)=\sum_{m=1}^{\infty} \frac{(4 m+1) I_{2 m} P_{2 m}(0)}{2 m(2 m+1) C_{r t} \frac{Q_{2 m}\left(\xi_{0}\right) P_{2 m}\left(\xi_{e}\right)-P_{2 m}\left(\xi_{0}\right) Q_{2 m}\left(\xi_{e}\right)}{Q_{2 m}^{\prime}\left(\xi_{0}\right) P_{2 m}\left(\xi_{e}\right)-P_{2 m}^{\prime}\left(\xi_{0}\right) Q_{2 m}\left(\xi_{e}\right)}-1} ; \\
& I_{2 m}=\int_{0}^{1} \ln (1+\eta) P_{2 m}(\eta) \mathrm{d} \eta, m=1,2, \ldots . .
\end{aligned}
$$

Thus, the fluid production rate is solely determined by the confocal coefficient $B_{0}$. From the property of $H\left(\lambda, \xi_{0}, \xi_{e}\right)$ and eqn. (12), it is observed that fluid production becomes infinitely large when the outer boundary approaches the inner boundary, $\xi_{e} \rightarrow \xi_{0}$. On the other hand, production becomes zero as the spheroid surface area shrinks to zero, $\xi_{0} \rightarrow 1$ (the spheroid degenerates to a line). On the contrary, for a two-dimensional fracture, the limit of very small width provides a non-zero fluid production as the fracture face area remains unchanged. 
The dimensionless fluid production rate

$$
\bar{Q}=\frac{\mu Q}{2 \pi \kappa_{s} \Delta p L}=\frac{\xi_{0}^{2}-1}{H\left(\lambda, \xi_{0}, \xi_{e}\right)}
$$

is a function of the permeability ratio $\lambda$ and the geometrical parameters $\xi_{0}, \xi_{e}$ only. Variation of $\bar{Q}$ with the permeability ratio $\lambda$ is shown in Fig. 2 for $\xi_{0}=1.005, \xi_{e}=2,5,10,50$. It is apparent that increasing the body-to-reservoir permeability ratio $\lambda$ increases the production rate. The rate of increase is more pronounced for $\lambda \leq 100$; and as the permeability ratio $\lambda$ becomes very large, $\bar{Q}$ quickly approaches its maximum value $(\lambda \rightarrow \infty)$.

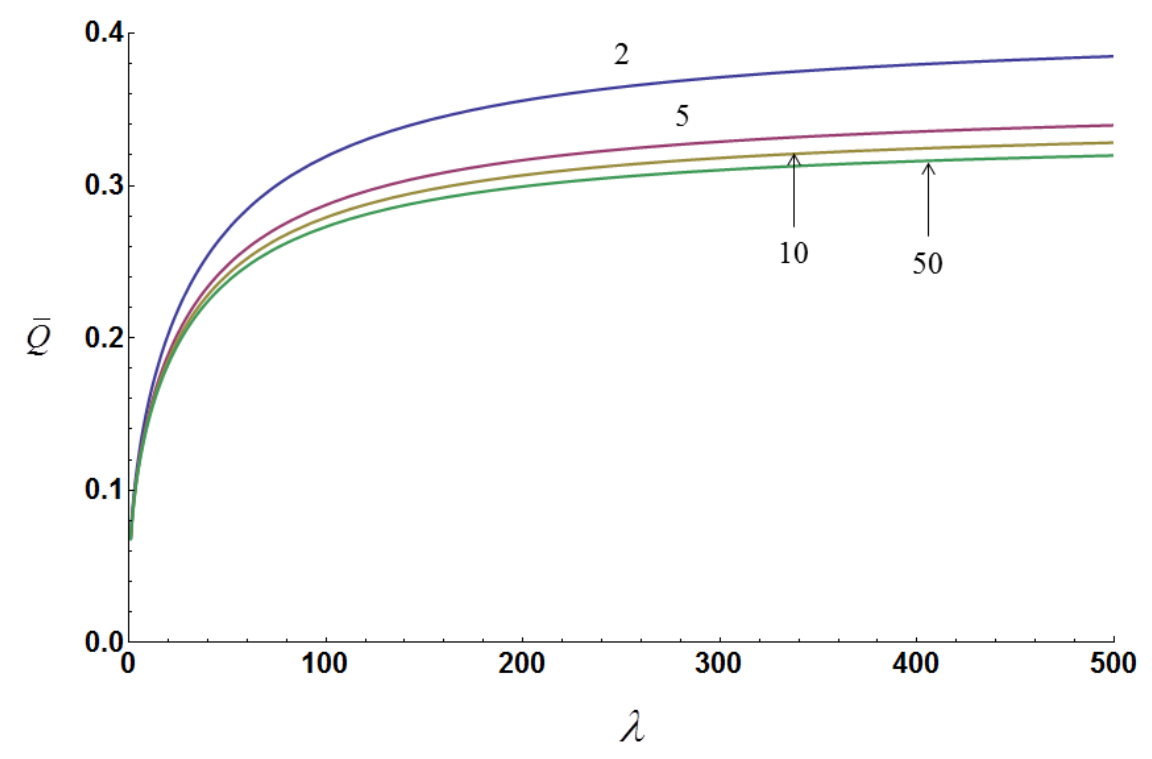

Fig. 2 Variation of $\bar{Q}$ with the permeability ratio $\lambda$ for $\xi_{0}=1.005$. The value of $\xi_{e}$ is marked above each curve.

When the permeability of the spheroid is much higher than the permeability of the reservoir, $\lambda \rightarrow \infty$, the pressure inside the semi-spheroid becomes a constant, and the fluid production rate is given by 


$$
Q=\frac{2 \pi \kappa_{s} L}{\mu} \frac{\Delta p}{Q_{0}\left(\xi_{0}\right)-Q_{0}\left(\xi_{e}\right)}=\frac{4 \pi \kappa_{s} L}{\mu} \frac{\Delta p}{\ln \frac{\xi_{0}+1}{\xi_{0}-1}-\ln \frac{\xi_{e}+1}{\xi_{e}-1}} .
$$

The radius at the base of the semi-spheroid is $r_{0}=L \sqrt{\xi_{0}^{2}-1}$ and the length of the semi-spheroid is $L \xi_{0}$. The fluid production rate given by the Gardner's single-root model [22] for a cylinder with the same radius and length, constant pressure inside the cylinder, and a cylindrical shell with an outer radius $r_{1}=L \sqrt{\xi_{e}^{2}-1}$ is

$$
Q_{G}=\frac{2 \pi \kappa_{s} L \xi_{0} \Delta p}{\mu} \frac{1}{\ln \left(r_{1} / r_{0}\right)} .
$$

Thus, the enhancement in the productivity due to the end effect can be measured by the ratio $Q / Q_{G}$

$$
\frac{Q}{Q_{G}}=\frac{2}{\xi_{0}} \frac{\ln \left(r_{1} / r_{0}\right)}{\ln \frac{\xi_{0}+1}{\xi_{0}-1}-\ln \frac{\xi_{e}+1}{\xi_{e}-1}},
$$

with

$$
\begin{aligned}
& \xi_{0}=\frac{l}{\sqrt{l^{2}-1}} \\
& \xi_{e}=\sqrt{1+\left(\frac{r_{1}}{r_{0}}\right)^{2} \frac{1}{l^{2}-1}} .
\end{aligned}
$$

$l$ is the semi-spheroid's length-to-base-radius ratio, $l=z_{0} / r_{0}$. The productivity ratio $Q / Q_{G}$ is plotted in Fig. 3 against the length-to-base-radius ratio $l$ and various values of $r_{1} / r_{0}$. The productivity ratio is always greater than one. Thus the end-effect always promotes production, similar to a two-dimensional fracture. This tip-induced production enhancement becomes more and more pronounced for shorter spheroid $\left(\operatorname{smaller} l\right.$ ). For example, for $r_{1} / r_{0}=80$, the 
production rate is increased by about $30 \%$ for $l=20$, while it is increased by $50 \%$ for a shorter spheroid with $l=10$. For very large length-to-radius ratios, the productivity ratios approach one from above. It is also observed that for larger fluid drawing volumes (larger $r_{1} / r_{0}$ ), the tipinduced production enhancement is also greater. Thus, the conventional constant-radius cylinder model without the end-effect significantly under-estimates the fluid extraction rate by a single root.

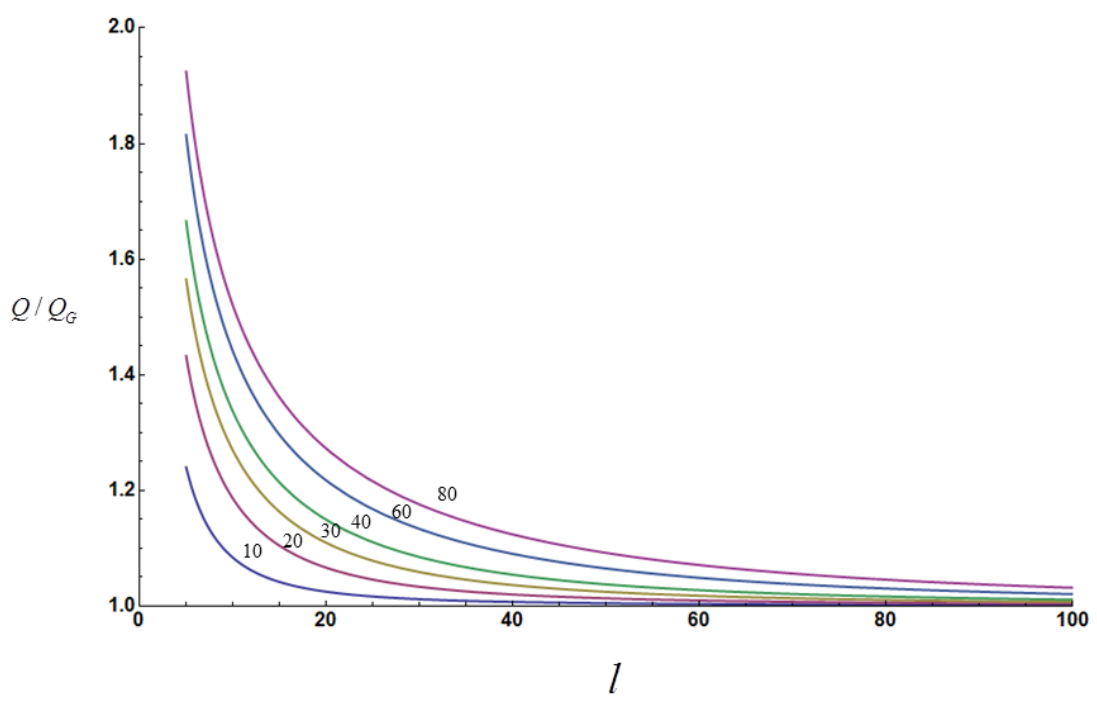

Fig. 3 Extraction rate ratio $Q / Q_{G}$ vs length-to-radius ratio. The values of $r_{1} / r_{0}$ are marked above each curve.

\section{Flux density distribution, reservoir flow decomposition, and fluid production} enhancement mechanism

The flux density of the fluid entering the spheroid through the spheroid-reservoir surface is

$$
q_{s}(\tilde{\eta})=\left.\left(-\mathbf{i}_{\tilde{\xi}}\right) \bullet \mathbf{v}(\tilde{\xi}, \tilde{\eta})\right|_{\tilde{\xi}_{0}}=\left.\frac{\kappa_{s}}{\mu} \frac{\sinh \tilde{\xi}_{0}}{L \sqrt{\sinh ^{2} \tilde{\xi}_{0}+\sin ^{2} \tilde{\eta}}} \frac{\partial p}{\partial \xi}\right|_{\xi_{0}} .
$$


Thus, the ratio between the flux density at the tip and that at the base is

$$
\frac{q_{\text {tip }}}{q_{\text {base }}}=O\left(1 / \tilde{\xi}_{0}\right)
$$

This tip-to-base flux density ratio becomes unbounded as $\tilde{\xi}_{0} \rightarrow 0$, indicating significantly higher flux density at the tip than that at the base as the tip becomes sharper. It can be shown that the flux density singularity is inversely proportional to the square-root of the distance to the tip, the same type of singularity as that for a two-dimensional fracture.

Further insights can be gained by analyzing the dimensionless flux density, which can be decomposed to:

$$
\begin{aligned}
& \bar{q}\left(\lambda, \tilde{\xi}_{0}, \tilde{\eta}\right)=\frac{\mu q_{s}(\tilde{\eta}) L}{\kappa_{s} \Delta p}=\bar{q}_{1}(\lambda, \tilde{\xi}, \tilde{\eta})+\bar{q}_{2}\left(\lambda, \tilde{\xi}_{0}, \tilde{\eta}\right) \\
& \bar{q}_{1}(\lambda, \tilde{\xi}, \tilde{\eta})=\frac{\sinh \tilde{\xi}_{0}}{\sqrt{\sinh ^{2} \tilde{\xi}_{0}+\sin ^{2} \tilde{\eta}}} \frac{1}{H\left(\lambda, \xi_{0}, \xi_{e}\right)}, \\
& \bar{q}_{2}\left(\lambda, \tilde{\xi}_{0}, \tilde{\eta}\right)=\frac{\sinh \tilde{\xi}_{0}}{\sqrt{\sinh ^{2} \tilde{\xi}_{0}+\sin ^{2} \tilde{\eta}}} \sum_{m=1}^{\infty}\left[\bar{A}_{2 m} P_{2 m}^{\prime}\left(\xi_{0}\right)+\bar{B}_{2 m} Q_{2 m}^{\prime}\left(\xi_{0}\right)\right] P_{2 m}(\eta), \\
& \bar{B}_{0}=\frac{B_{0}}{\Delta p}=-\frac{\xi_{0}^{2}-1}{H\left(\lambda, \xi_{0}, \xi_{e}\right)}, \bar{A}_{2 m}=\frac{A_{2 m}}{\Delta p}, \bar{B}_{2 m}=\frac{B_{2 m}}{\Delta p}
\end{aligned}
$$

The dimensionless surface flux density $\bar{q}$ and its two contributors $\bar{q}_{1}, \bar{q}_{2}$ are shown in Fig. 4-6, for different values of the permeability ratio $\lambda=50,100,150,200$, with $\xi_{0}=1.005, \xi_{e}=50$. Fig. 4 shows the flux density $\bar{q}_{1}$, which is produced by the confocal spheroidal flow (the term with $\bar{B}_{0}$ ) in the reservoir. Clearly, this flux density has a minimum at the base, and a maximum at the tip which is nearly singular. Increasing the permeability ratio $\lambda$ increases the flux density $\bar{q}_{1}$ everywhere. However the rate of this increase diminishes as $\lambda$ increases to larger 
values; and when the permeability ratio $\lambda$ goes beyond $150, \bar{q}_{1}$ has nearly reached its limiting value.

The flux density contributor $\bar{q}_{2}$ is shown in Fig. 5, for the same set of parameters used for $\bar{q}_{1}$. This flux density contribution is due to the non-confocal components of the flow in the reservoir, $A_{2 m}, B_{2 m}, m \geq 1 . \bar{q}_{2}$ experiences a sign change, from positive values near the base to negative values near the tip. The change of sign occurs around $\eta \approx x \approx 0.4 . \bar{q}_{2}$ near the tip is also nearly singular. A very important feature of the flux density $\bar{q}_{2}$ is that it does not contribute to the net fluid production, even though the area under $\bar{q}_{2}$ is not zero. This is due to the nature of the curvilinear coordinate: the dimensionless fluid production rate is given by the weighted integral

$$
\bar{Q}=\int_{0}^{\pi / 2} \bar{q}\left(\tilde{\xi}_{0}, \tilde{\eta}\right) \sinh \tilde{\xi}_{0} \sin \tilde{\eta} \sqrt{\sinh ^{2} \tilde{\xi}_{0}+\sin ^{2} \tilde{\eta}} d \tilde{\eta}=\sqrt{\xi_{0}^{2}-1} \int_{0}^{1} \bar{q}\left(\xi_{0}, \eta\right) \sqrt{\xi_{0}^{2}-\eta^{2}} d \eta
$$

which is not the area under the $\bar{q}$ curve. Another outstanding feature for $\bar{q}_{2}$ is that it is significantly reduced as the permeability ratio $\lambda$ is increased. 


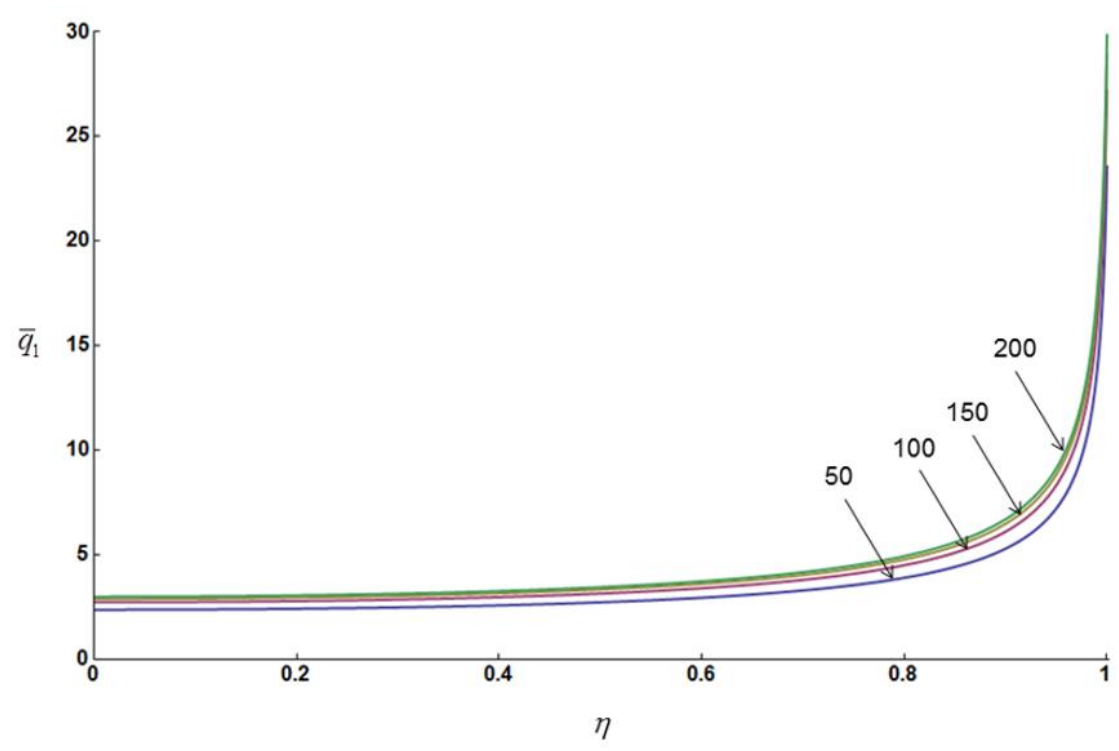

Fig. 4 Flux density contributor $\bar{q}_{1}$ for $\lambda=50,100,150,200$ (marked), with $\xi_{0}=1.005, \xi_{e}=50$.

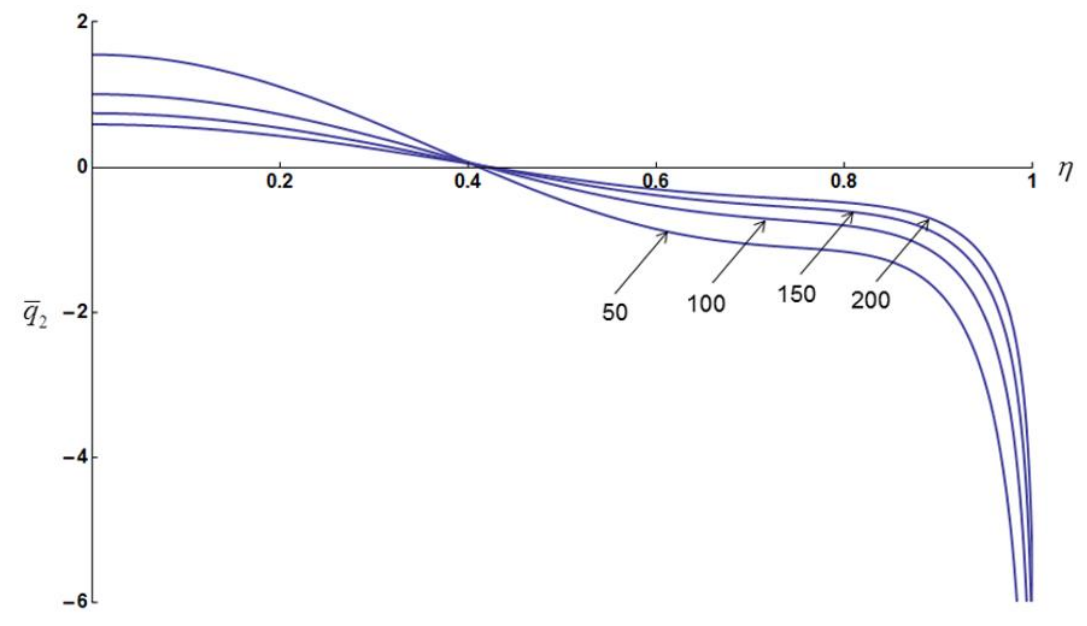

Fig. 5 Flux density contributor $\bar{q}_{2}$ for $\lambda=50,100,150,200$ (marked), with $\xi_{0}=1.005, \xi_{e}=50$. 


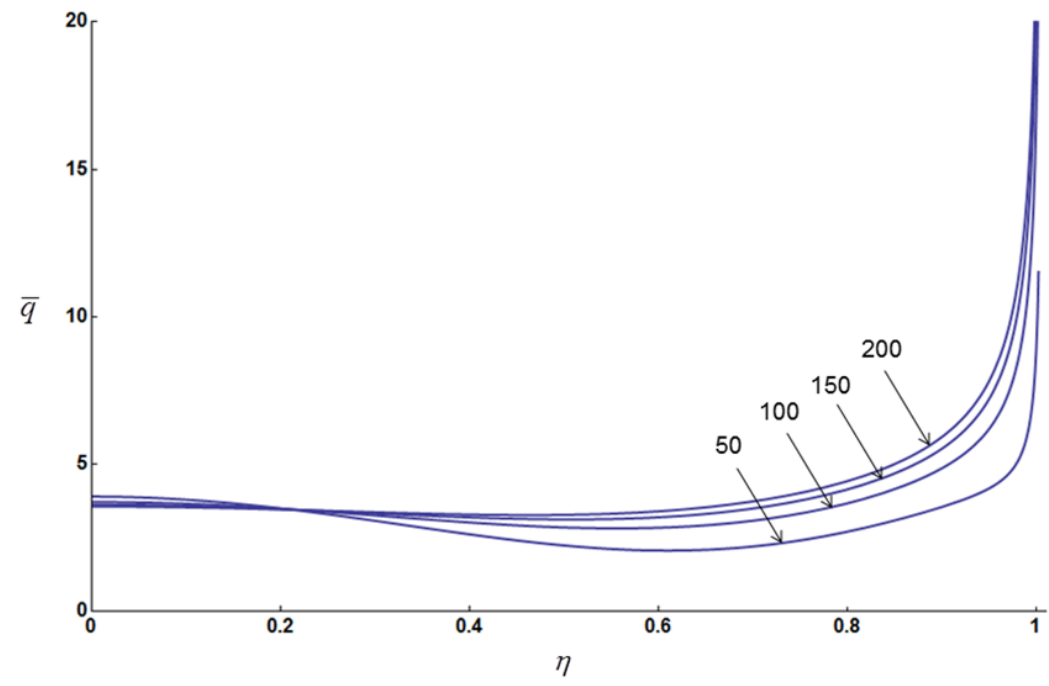

Fig. 6 Flux density contributor $\bar{q}$ for $\lambda=50,100,150,200$ (marked), with $\xi_{0}=1.005, \xi_{e}=50$.

The flux density $\bar{q}$ is the sum of $\bar{q}_{1}$ and $\bar{q}_{2}$, and it is shown in Fig. 6. While $\bar{q}$ increases as the permeability ratio $\lambda$ goes up, the resulting increase in the fluid production-rate $\bar{Q}$ is not due to a decrease in $\bar{q}_{2}$, since $\bar{q}_{2}$ does not contribute to fluid production regardless the value of the permeability ratio. Rather, this increase in $\bar{Q}$ is caused solely by the increase in $\bar{q}_{1}$, as shown in Fig. 4.

It should be emphasized the flux density distribution on the spheroid-reservoir surface $q_{s}(\tilde{\eta})$ is not the same as the axial flux inside the spheroid, $q_{r}(\tilde{\eta})$. They are related by eqn. (4). However, $q_{r}(\tilde{\eta})$ is always zero at the spheroid tip and it increases monotonically as we move toward the center of the spheroid. It is also noteworthy that even though the pressure gradient at the tip is nearly singular, the pressure at the tip remains finite and well above zero. Thus, there is no cavitation in the reservoir near the tip. 
The velocity field in the reservoir can also be decomposed into a confocal spheroidal flow and a distributive flow:

$$
\overline{\mathbf{v}}(\tilde{\xi}, \tilde{\eta})=\frac{\mu L \mathbf{v}(\tilde{\xi}, \tilde{\eta})}{\kappa_{s} \Delta p}=\mathbf{i}_{\tilde{\xi}} \overline{\mathbf{v}}_{\text {conf }}+\overline{\mathbf{v}}_{\text {dist }}(\tilde{\xi}, \tilde{\eta})
$$

The confocal spheroidal component is solely determined by the coefficient $\bar{B}_{0}$ :

$$
\overline{\mathrm{v}}_{\text {conf }}=\frac{\sinh \tilde{\xi}}{\sqrt{\sinh ^{2} \tilde{\xi}+\sin ^{2} \tilde{\eta}}} \bar{B}_{0} Q_{0}^{\prime}(\xi)=\frac{\sinh \tilde{\xi}}{\sqrt{\sinh ^{2} \tilde{\xi}+\sin ^{2} \tilde{\eta}}} \frac{1}{H\left(\lambda, \xi_{0}, \xi_{e}\right)} .
$$

The confocal flow field, the redistributive flow field, and the net flow field $\overline{\mathbf{v}}_{\text {conf }}, \overline{\mathbf{v}}_{\text {dist }}, \overline{\mathbf{v}}$ are shown in Fig. 7-9, for $\lambda=50, \xi_{0}=1.005, \xi_{e}=50$. Only the confocal flow is fluid-producing, while the redistributive flow simply moves fluid in and out of the spheroid. The net flow shows a converging and focusing flow pattern near the spheroid tip, just like the confocal flow. This flow pattern is the reason that the flux density exhibits a nearly singular behavior near the tip, and it is also the mechanism that enhances fluid production as compared to models without an end effect, such as the Gardner's model discussed in the previous section. As the permeability ratio $\lambda$ is increased, the strength of the confocal flow increases while the srength of the distributive flow decreases. When $\lambda \rightarrow \infty$, the distributive flow vanishes, and the reservoir flow becomes purely confocal. 


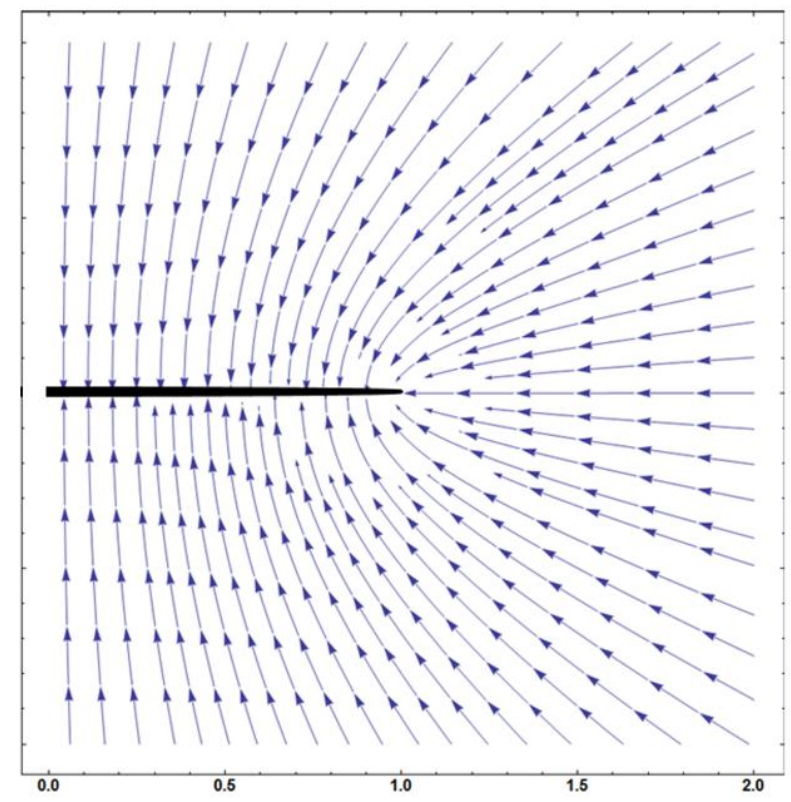

Fig. 7 The confocal flow field. Only half of the spheroid is shown.

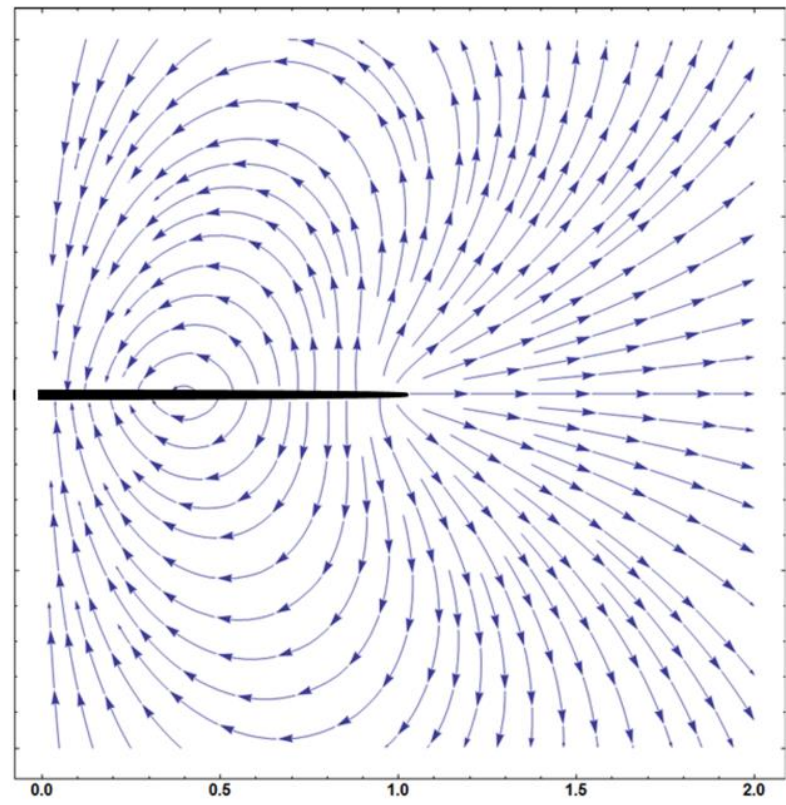

Fig. 8 The redistributive flow field. 


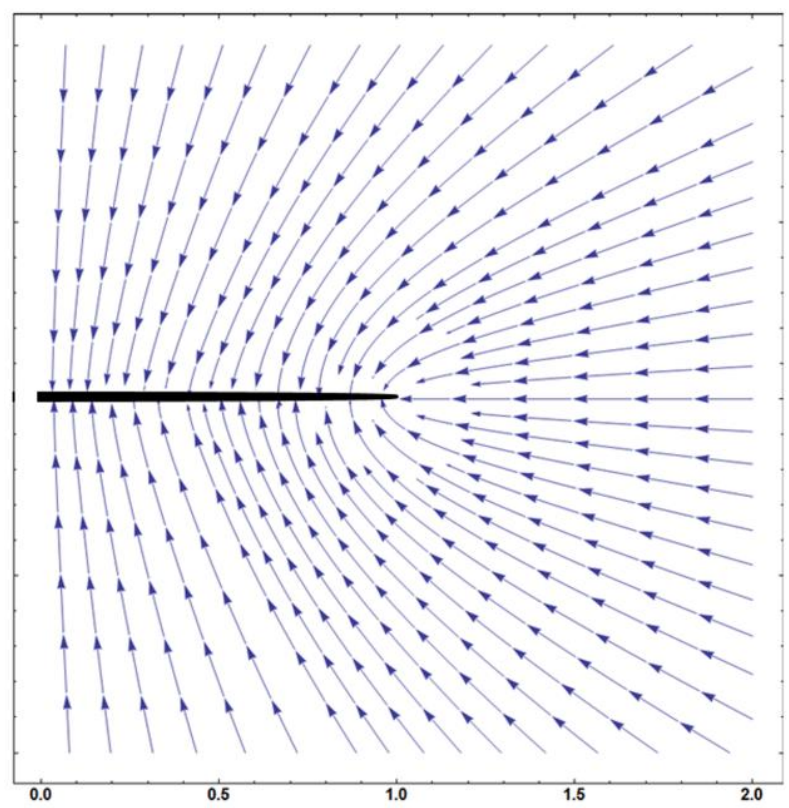

Fig. 9 The net flow field.

\section{Shape optimization for maximum fluid production}

The volume of a prolate semi-spheroid is given by

$$
V=\frac{2}{3} \pi x_{0} z_{0}^{2}
$$

and the fluid production rate can be expressed as

$$
Q=\frac{2 \pi \kappa_{s}}{\mu}\left(\frac{3 V}{2 \pi}\right)^{1 / 3} \Delta p J\left(\lambda, \xi_{0}, \xi_{e}\right)
$$

with

$$
J\left(\lambda, \xi_{0}, \xi_{e}\right)=\frac{\left(\xi_{0}^{2}-1\right)^{2 / 3}}{\xi_{0}^{1 / 3}} \frac{1}{H\left(\lambda, \xi_{0}, \xi_{e}\right)}
$$


Thus, when the pressure drop, body volume and permeability ratio are prescribed, maximizing the dimensionless shape-dependent function $J\left(\lambda, \xi_{0}, \xi_{e}\right)$ maximizes the fluid production rate. The optimization can be performed with respect to the length-to-base-radius ratio $l$ :

$$
l=\frac{z_{0}}{x_{0}}=\frac{\xi_{0}}{\sqrt{\xi_{0}^{2}-1}} .
$$

In Fig. 10, the shape function $J$ is plotted against the length-to-base-radius ratio $l$, for $\xi_{e}=50$. The values of the permeability ratio $\lambda$ are marked above each curve. When $\xi_{0} \rightarrow 1$, the spheroid body degenerates to an infinitely long line, $l \rightarrow \infty$, and the spheroid surface area goes to zero. Thus, the fluid production rate becomes zero. On the other hand, for any given outer boundary location $\xi_{e}$, (30) indicates that as the length-to-base-radius ratio $l$ decreases, $\xi_{0}$ increases. Continued decrease in $l$ can lead to $\xi_{0} \rightarrow \xi_{e}$, with the inner and outer boundaries coincide with each other. This causes the production rate to become unbounded. This unbounded production rate limit manifests itself for small $l$ in Fig. 10. Of course this limit may have already violated the slenderness assumption, which requires $l$ to be relatively large. In the range of $l>>1$, for any permeability ratio $\lambda \geq 30$, there exists a length-to-base-radius ratio $l_{m}$ that creates a local maximum in the fluid production rate. This optimum length-to-radius ratio $l_{m}$ is plotted in Fig. 11 for $\xi_{e}=5,20,50$. The change in $l_{m}$ with $\xi_{e}$ is relatively small. However, for smaller $\xi_{e}, l_{m}$ does not exist for smaller permeability ratios. For example, when $\xi_{e}=5, l_{m}$ exists only when $\lambda \geq 50$. 


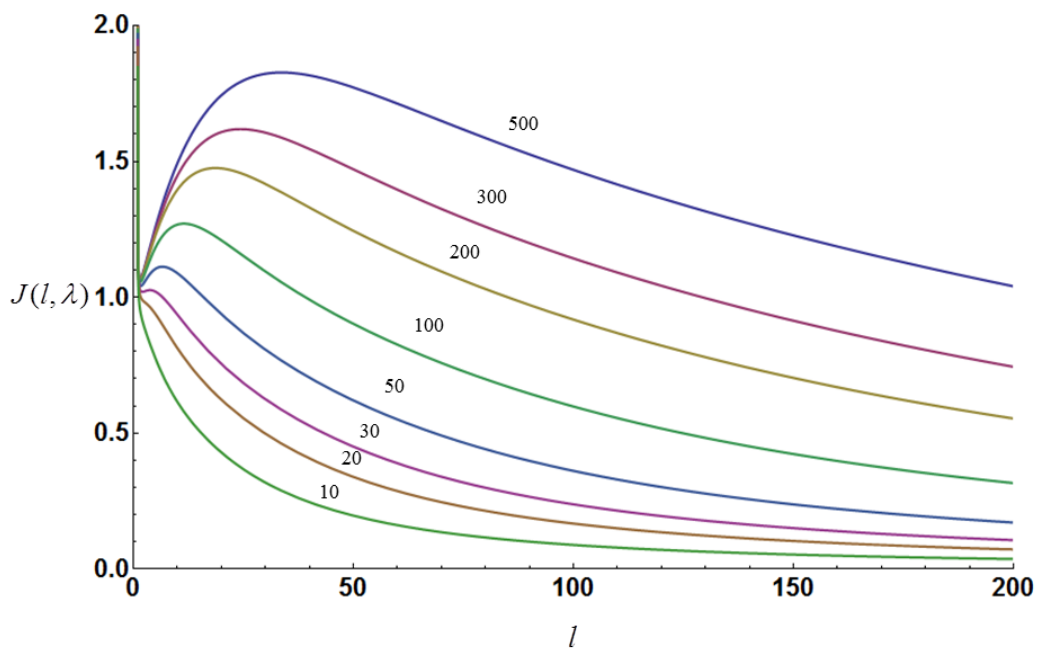

Fig. 10 Variation of $J$ as a function of the length-to-base-radius ratio $l$ with the permeability ratio $\lambda$ as a parameter (marked above each curve). $\xi_{e}=50$.

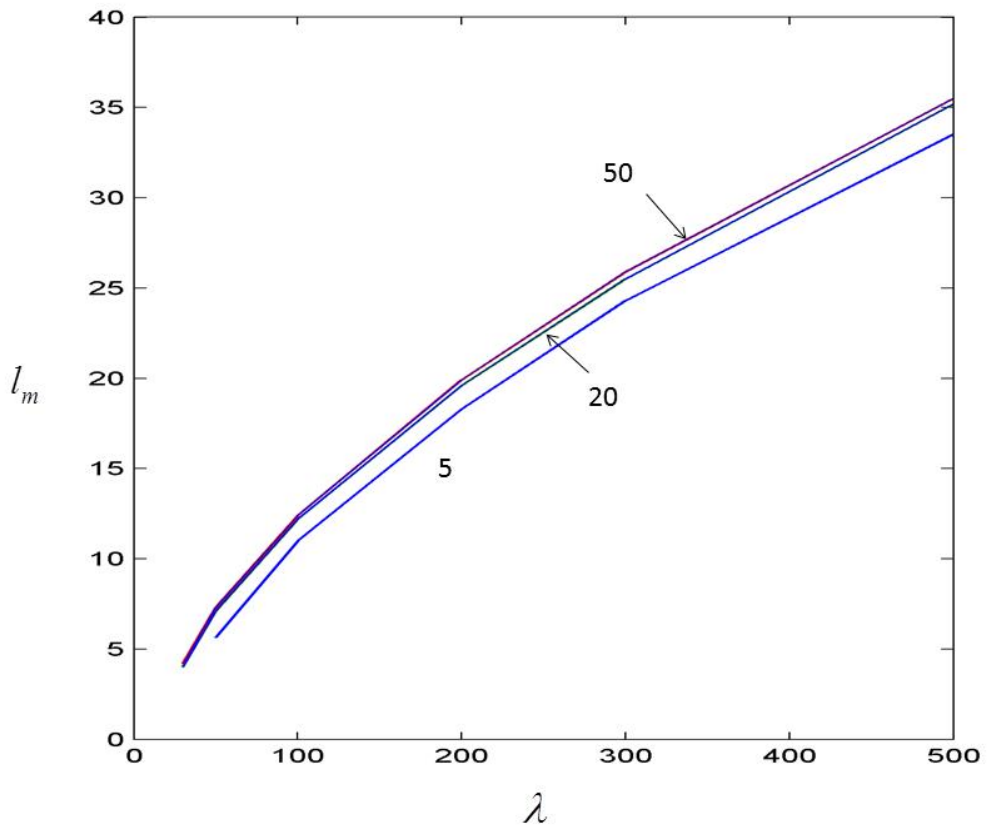

Fig. 11 Optimum length-to-base-radius ratio $l_{m}$ vs the permeability ratio $\lambda$. The value of $\xi_{e}$ are marked on each curve. 


\section{Conclusions}

Fluid extraction from a porous medium by a slender permeable prolate-spheroid is studied in this paper with a focus on the effect of the body tip on the fluid production enhancement. It is found that the pressure gradient of the reservoir flow at the tip is nearly singular for a slender spheroid, and the flow converges and focuses to the tip. As a result, fluid production is significantly higher than that predicted by the infinitely-long cylinder model frequently used in the literature. Furthermore, for a fixed spheroid body volume, there exists an optimum length-toradius ratio that maximizes fluid production, provided the spheroid-to-reservoir permeability ratio is sufficiently large. The results obtained in this study can serve as the basis to construct more sophisticated mesoscopic single-root models for simulating water-uptake by a plant's root system.

Acknowledgement This work is supported by the National Science Foundation and the Donors of the Petroleum Research Fund, administered by the American Chemical Society.

Appendix The Coefficients $A_{2 m}, B_{2 m}$

The coefficients $A_{2 m}, B_{2 m}$ are used in Sections 3-5 to evaluate the pressure and related quantities.

$\Delta p=p_{e}-p_{w} ; B_{0}=-\frac{\xi_{0}^{2}-1}{H\left(\lambda, \xi_{0}, \xi_{e}\right)} \Delta p ; A_{0}=p_{e}-B_{0} Q_{0}\left(\xi_{e}\right)$

$m \geq 1$ :

$B_{2 m}=\frac{2 m(2 m+1)(4 m+1) I_{2 m} Q_{0}^{\prime}\left(\xi_{0}\right) P_{2 m}\left(\xi_{e}\right)}{2 m(2 m+1) C_{r t}\left[Q_{2 m}\left(\xi_{0}\right) P_{2 m}\left(\xi_{e}\right)-P_{2 m}\left(\xi_{0}\right) Q_{2 m}\left(\xi_{e}\right)\right]-\left[Q_{2 m}^{\prime}\left(\xi_{0}\right) P_{2 m}\left(\xi_{e}\right)-P_{2 m}^{\prime}\left(\xi_{0}\right) Q_{2 m}\left(\xi_{e}\right)\right]} B_{0}$ $A_{2 m}=-B_{2 m} \frac{Q_{2 m}\left(\xi_{e}\right)}{P_{2 m}\left(\xi_{e}\right)}$ 


\section{Reference}

[1] M. J. Economides, T. Martin, Modern Fracturing: Enhancing Natural Gas Production, Energy Tribune Publishing, Houston, 2007.

[2] K. P. Chen, Y. Jin, M. Chen, Pressure-gradient singularity and production enhancement for hydraulically-fractured wells, Geophysical J. Intl. 195(2013) 923-931.

[3] Y. Jin, K. P. Chen, M. Chen, Analytical solution and mechanisms of fluid production from hydraulically fractured wells with finite fracture conductivity. J. Eng. Math. 92(2015) 103-122.

[4] M. A. Zwieniecki, M. V. Thompson, N. M. Holbrook, Understanding the hydraulics of porous pipes: tradeoffs between water uptake and root length utilization, J. Plant Growth Regul.,21(2003) 315-323.

[5] J. B. Passioura, Water Transport in and to roots, Ann. Rev. Plant Physiol. Plant Mol. Biol. 39(1988), 245-265.

[6] T. Roose, A. C. Fowler, A model for water uptake by plant roots, J. Theor. Bio. 228(2004), 155-177.

[7] P.A.C. Ratts, Uptake of water from soils by plant roots, Transp. Porous Med. 68, 5 (2007).

[8] T. Roose, A. Schnepf, Mathematical models for plant-soil interaction, Phil. Trans. R. Soc. A, 366(2008) 4597-3611.

[9] F. J. Molz, Models of water transport in the soil-plant system, Water Resour. Res., 17(1981) 1245-1260. 
[10] C. Doussan, G. Vercambre, L. Pages, Modeling of the hydraulic architecture of root systems: an integrated approach to water absorption-distribution of axial and radial conductances in maize, Ann. Bot., 81(1998) 225-232.

[11] V. Clausnitzer, J. V. Hopmans, Simultaneous modeling of transient three-dimensional root growth and soil water flow, Plant Soil, 164(1994) 299-314.

[12] J. R. Philip, The physical principles of soil water movement during the irrigation cycle, In: Proc. Intern. Congr. Irrigation and Drainage, ${ }^{\text {rd }}$, San Francisco, 8(1957) 125-154 .

[13] W. R. Gardner, Dynamic aspects of water availability to plants, Soil Sci., 89(1960) 63-73.

[14] I. R. Cowan, Transport of water in the soil-plant-atmosphere system, J. Appl. Ecol. 2(1965) 221-239.

[15] J. J. Landsberg, N. D. Fowkes, Water movement through plant roots, Ann. Bot., 42(1978) 493-508.

[16] J. M. Hainsworth, L.A.G. Aylmore, Water extraction by single plant roots, Soil Sci. Soc. Am. J. 50(1986) 841-848. 